\title{
Utilizing Kinematics and Selective Sweeping in Reinforcement Learning-Based Routing Algorithms for Underwater Networks
}

\author{
R. Plate ${ }^{a, *}$, C. Wakayama ${ }^{a}$ \\ ${ }^{a}$ Maritime Systems Division, SPAWAR Systems Center Pacific, Code 56490, 53560 Hull St., San Diego, CA \\ 92152-5001, USA
}

\begin{abstract}
Effective utilization of mobile ad hoc underwater distributed networks is challenging due to high system costs and the harsh environment characterized by low bandwidth, large latency, high energy consumption, and node mobility. This work addresses the routing issue, which is critical in successfully establishing and utilizing an underwater network. In particular, it focuses on reinforcement learning $(\mathrm{RL})$-based routing algorithms, which possess the ability to explore the network environment and adapt routing decisions to the constantly changing topology of the network due to node mobility and energy usage. This paper presents a routing algorithm based on Q-learning, one of the RL approaches, with additional Kinematic and Sweeping features, therefore referred to as QKS. These two additional features are introduced to address the potential slow convergence associated with pure RL algorithms. The results of a detailed packet-level simulation have been obtained using the NS-2 open-source network simulator with underwater modeling additions. The energy efficiency, convergence, and delivery performance of QKS are compared with two other routing protocols for underwater networks, a basic flooding approach (ICRP (Liang, 2007)) and a basic Q-learning implementation (QELAR (Hu, 2010)), using simulations of networks with both fixed and mobile nodes.
\end{abstract}

Keywords: reinforcement learning, routing protocol, acoustic communication, underwater sensor network

${ }^{*}$ Corresponding Author. Phone: +1 (619) 553-2076

Email addresses: rplate@spawar.navy.mil (R. Plate), wakayama@spawar.navy.mil (C. Wakayama) 


\section{Introduction and Related Work}

Given the advances in underwater transmission capabilities, underwater wireless networking has emerged as an active research area with applications in a wide range of scientific and military domains $[1,2,3]$. The physical limitations of the underwater acoustic environment characterized by long propagation delay, low bandwidth, high energy consumption and node mobility prevent the direct use of conventional routing protocols developed for radio frequency (RF) ad hoc networks. These factors must be carefully taken into account in designing wireless underwater acoustic networks.

Conventional routing protocols developed for RF ad hoc networks can be classified into three basic categories: table-based proactive, on-demand reactive, and geographical [4]. Each of these has inherent weaknesses when applied in underwater acoustic environments. Proactive routing has high maintenance costs for storing information about neighbor nodes every time there is a topology change, while reactive routing has high route discovery costs to gather neighbor information as it is needed. Geographic routing relies on position and/or velocity information, which can be difficult to obtain or inaccurate depending on node capabilities. Combining the strengths of these three can produce a more robust routing approach adequate for underwater applications.

Many existing routing protocols for underwater networks address particular underwater challenges and depend on limiting application scenarios. A recent survey on routing protocols for underwater acoustic wireless sensor networks can be found in [5]; we present a subset of the existing protocols according to their special features addressing node mobility and energy efficiency. Vector-based-forwarding (VBF), depth-based-routing (DBR) and mobicast routing (MR) protocols address the challenges due to node mobility. The VBF protocol [6] establishes a routing pipe between the source and destination, and only the nodes in the routing pipe can participate in message forwarding. The positions of the source and the destination are assumed available in defining a routing vector between the source and the destination. In DBR [7], each node accounts for its depth and the depth of the previous node, and uses a greedy algorithm to forward packets only along the upward directions. The MR [8] protocol uses predefined routes for autonomous underwater vehicles (AUVs) for gathering data in a predefined sensor network. A dedicated AUV is controlled to follow a specified route to sequentially service nodes that are allocated into three-dimensional zones of reference (3D-ZOR); nodes in adjacent 3D-ZOR must be woken up to be queried while 
trying to avoid topology holes due to node drift by the ocean current.

Limited battery energy is a fundamental issue for underwater nodes and many routing efforts focus on energy efficiency and network lifetime. Focused beam routing (FBR) [9] is a cross-layer approach in which the routing protocol, the medium access control and the physical layer functionalities are coupled by power control to establish energy efficient routes dynamically. In FBR, the positions of both the source and destinations must be known. Energy-efficient routing protocol (EUROP) described in [10] is designed to reduce broadcasting hello messages by equipping each node with pressure sensing capability. Each node calculates its depth, determines its corresponding layer, and selects the next node by applying a deep to shallow routing rule; it is assumed that the destination node is located on the water surface. Another Energy-efficient Routing Protocol based on Physical distance and Residual energy $\left(\mathrm{ERP}^{2} \mathrm{R}\right)[11]$ takes into account the physical distances of the nodes towards the destination and the residual energy of the nodes to determine the next forwarder in order to extend the network lifetime. The information-carrying-based routing protocol (ICRP) [12] is a reactive protocol designed to provide energy efficient, real-time and scalable routing based on a limited flooding approach. Due to the high overhead of finding new routes as they change, ICRP may not be appropriate for underwater networks consisting of high mobility nodes, yet is attractive due to its simplicity.

The underwater challenges and limited applications of existing underwater routing protocols demand robust, adaptive, and energy-efficient routing approaches which require minimum a priori knowledge about the network and restrictions on network architecture. These requirements motivate the development of underwater routing protocols based on machine learning techniques. Multi-agent machine learning techniques, such as Multi-Agent Reinforcement Learning (MARL), have been applied successfully in many problem domains involving distributed decision making in which each agent must learn by interacting with its dynamic environment and attempt to discover optimal actions on their own [13]. A fully distributed underwater routing problem can be seen as a collaborative multi-agent system. In [14], a commonly used machine learning technique known as Q-learning is implemented in a routing algorithm called QELAR to address a fully distributed architecture. Nodes compute their own routing decisions by storing routing information (Q-values) of their direct neighbor nodes. In QELAR, the Q-value estimates consider the energy consumption of sensor nodes and residual energy distribution among neighboring nodes to optimize total energy 
consumption and network lifetime. The environment is learned as the estimated Q-values converge to reflect the network topology. However, convergence could occur slowly for certain network configurations such as those comprised of a large number of nodes and/or nodes with high mobility, resulting in excess resource usage. A multi-level routing protocol (MURAO) [15] is a two-layer Q-learning based routing protocol which is designed for dense, hybrid acoustic-optical underwater sensor networks. In MURAO, a cluster-based two layer network is formed, where neighboring clusters of nodes share at least one common gateway node. A cluster head is associated with each cluster and directs inter-cluster routing, while the individual nodes of the clusters perform intra-cluster routing. The slow convergence of Q-learning associated with a dense network is partly addressed by allowing routing in different clusters to take place in parallel.

In this paper, we investigate two additional features that can improve the convergence rate of a basic Q-learning algorithm and its ability to track changes in the network while balancing the routing overhead. The first is the use of kinematic information to add a geographic component to the RL approach. Nodes transmit their own position and velocity estimates and store those of their neighbors to enable more accurate estimation of successful transmission probability, resulting in fewer failed transmissions. We assume that a node can estimate its own position and velocity (either independently or collaboratively) but is unaware of the destination location. The second is the addition of selective backward exploration (sweeping) to the forward exploration of QELAR, such that nodes actively propagate significant changes in their Q-values back toward the source. Although overhead cost is increased with the addition of each feature, the improved convergence rate and tracking of network changes results in an overall improvement in energy consumption and/or latency as compared to the baseline Q-routing approach. We will refer to our algorithm incorporating these features as QKS (Q-learning utilizing Kinematics and Sweeping). Our objective is to investigate the performance of RL techniques as applied to routing in underwater networks and evaluate how the two additional features developed can improve performance and address certain weaknesses in a more basic Q-learning implementation. Detailed packet-level simulations are run to understand the trade-offs achievable when employing these algorithms in different network scenarios so that the best features/parameter values can be chosen for maximal efficiency.

This paper proceeds as follows. In Section 2, we introduce Q-learning and a baseline algorithm, QELAR. In Section 3 we discuss the two unique features of the QKS algorithm - utilizing kinematic 
information and selective sweeping - which we have developed to improve the convergence rate. We also briefly describe the ICRP algorithm in Section 4 which we use for comparison in our simulations. Section 5 presents NS-2 [16] simulation results that demonstrate the performance of the two additional features; the energy usage, convergence, and delivery performance results are compared to two baseline algorithms: ICRP (simple flooding-based approach) and QELAR (basic $\mathrm{RL}$ approach). Conclusions and future work are provided in Section 6.

\section{Q-Learning-Based Routing Algorithms}

The sequential routing decision system can be modeled as a Markov Decision Process (MDP). The MDP is characterized by its state set, action set, dynamics (set of state transition probabilities), and expected immediate reward. Let $s_{t}$ and $a_{t}$ denote a state and action (decision), respectively, at time $t$; for the underwater network system, the state represents the location of the packet in the network and the action represents which of the one-hop neighbor nodes of $s$ to forward to. Let $P_{s_{t} s_{t+1}}^{a_{t}}$ denote the probability of going from the current state, $s_{t}$, to the next state, $s_{t+1}$, when taking action $a_{t} ; R_{s_{t} s_{t+1}}^{a_{t}}$ is the expected immediate reward for taking action $a_{t}$ at state $s_{t}$ and arriving at state $s_{t+1}$. Reward may include components related to energy usage, network lifetime, delay, and congestion depending on the mission operational objectives. The objective is to maximize the expected sum of rewards of actions leading from the source node to the destination node. According to Bellman's principle of optimality in Dynamic Programming, the optimal solution can be obtained once the optimal value functions are found [17]. Value functions are functions of the states that provide a measure of how good it is to be in a given state $(V)$, or of state-action pairs that estimate how good it is to perform a given action in a given state $(Q)$. Optimal value functions, $V^{*}$ and $Q^{*}$, satisfy the Bellman equations [17]:

$$
\begin{aligned}
V^{*}\left(s_{t}\right) & =\max _{a_{t}} \sum_{s_{t+1}} P_{s_{t} s_{t+1}}^{a_{t}}\left[R_{s_{t} s_{t+1}}^{a_{t}}+\gamma V^{*}\left(s_{t+1}\right)\right], \\
Q^{*}\left(s_{t}, a_{t}\right) & =\sum_{s_{t+1}} P_{s_{t} s_{t+1}}^{a_{t}}\left[R_{s_{t} s_{t+1}}^{a_{t}}+\gamma \max _{a_{t+1}} Q^{*}\left(s_{t+1}, a_{t+1}\right)\right],
\end{aligned}
$$

where $0 \leq \gamma \leq 1$ is a discount parameter that determines how important future rewards are. The relation between $V^{*}$ and $Q^{*}$ is given by:

$$
V^{*}\left(s_{t}\right)=\max _{a_{t}} Q^{*}\left(s_{t}, a_{t}\right) .
$$


Value functions evaluated at given states are referred to as V-values, while functions evaluated as state-action pairs are referred to as Q-values. For mobile ad hoc underwater networks, where the system model of future behavior is not known at each node, the optimal value functions are not known a priori, and also change as the network changes. In this situation, methods of estimating the optimal value functions must be employed to find good routing decisions. Q-learning is a reinforcement learning method which iteratively approximates $Q^{*}$.

\subsection{QELAR Algorithm}

QELAR is a recently-developed routing algorithm for underwater sensor networks that implements a model-based Q-learning approach [14]. The Q-values represent the estimated reward (based on energy usage) for a packet to reach the destination node from the neighboring nodes. Nodes approximate Eq. (2) to evaluate neighbor node Q-values and choose the neighbor with the maximum Q-value (Eq. (3)). The reward function considers the initial node energy, residual energy, and the energy distribution of the neighboring nodes to balance choosing short paths with maximizing network lifetime. Each time a node makes a routing decision, there are two possible outcomes that can occur: the action is successful and the state transitions to $s_{t+1}$, or the action fails and the state remains at the initial state, $s_{t}$. The reward function in the former case is given by

$$
R_{s_{t} s_{t+1}}^{a_{t}}=-g-\alpha_{1}\left[c\left(s_{t}\right)+c\left(s_{t+1}\right)\right]+\alpha_{2}\left[d\left(s_{t}\right)+d\left(s_{t+1}\right)\right]
$$

where $g$ is a constant cost accounting for the energy used in transmitting. The $c(s)$ terms provide an increasing penalty for transmitting to a node as that node's ratio of energy available to initial energy decreases, and $d(s)$ provides a second penalty based on how much energy that node has relative to its neighbors; $\alpha_{1}$ and $\alpha_{2}$ are weighting parameters. The reward for the failure case is given by:

$$
R_{s_{t} s_{t}}^{a_{t}}=-g \eta-\beta_{1} c\left(s_{t}\right)+\beta_{2} d\left(s_{t}\right)
$$

where $\beta_{1}$ and $\beta_{2}$ are separate weighting parameters. In (5), an additional weight, $\eta>1$, is introduced to scale $g$ to account for the fact that a failed transmission will have not only its own transmission cost, but will require a retransmission and therefore incur additional future costs. This is a modification from the QELAR implementation presented in [14] to improve performance in the case where a node estimates all of its neighbors to be undesirable (have lower V-values) 
as next hops (e.g. due to inaccurate $\mathrm{V}$-value estimates); with $\eta=1$ (original version), the node would tend to choose the neighbor with the lowest probability of successful transmission because it calculated that a failure was better than succeeding to any neighbor - certainly not a beneficial choice. The addition of $\eta$ provides a penalty for failing above and beyond the penalty of merely remaining at the current state.

The reward for success and the reward for failure can be combined using the probabilities of each case occurring to produce an estimated reward for that action:

$$
r_{t}=P_{s_{t} s_{t+1}}^{a_{t}} R_{s_{t} s_{t+1}}^{a_{t}}+P_{s_{t} s_{t}}^{a_{t}} R_{s_{t} s_{t}}^{a_{t}}
$$

and, based on equation (2), the $\mathrm{Q}$ function can then be written:

$$
Q\left(s_{t}, a_{t}\right)=r_{t}+\gamma\left[P_{s_{t} s_{t+1}}^{a_{t}} V\left(s_{t+1}\right)+P_{s_{t} s_{t}}^{a_{t}} V\left(s_{t}\right)\right] .
$$

Transition probabilities to neighbors are estimated based on the success/failure history of a node's forwarding actions.

When nodes overhear neighboring forwarding transmissions, they use the information obtained to update estimates of their neighbors' V-values. In order to keep up with changing network topology, nodes also periodically transmit header-only packets with their V-value and energy information to neighbors if they have not transmitted within a specified period of time. Thus, a combination of virtual experiments (calculated using equation (7)) and actual observation (overhearing neighbor transmissions) are employed to make routing decisions.

\section{The QKS Algorithm}

For networks comprised of nodes with high mobility, QELAR requires frequent periodic pinging to allow nodes to update their $\mathrm{P}$ and $\mathrm{V}$ estimates; inaccurate estimates result in many transmission failures and therefore energy waste and potential network flooding. Additionally, a significant number of forwarding actions is required to accurately estimate the probability of successful transmission simply based on the history of which ones failed. This can lead to slow convergence, which is particularly problematic if the network is changing quickly. As an approach to mitigate these issues and improve the ability to predict state transition probabilities and enhance V-estimate convergence, we discuss the utilization of neighbor kinematic data (position and velocity) and selective sweeping as employed by our modified Q-learning algorithm, QKS. 


\subsection{Utilizing Kinematics}

Although QELAR's scheme of learning transition probabilities from the success/failure rate of its history of forwarding actions can be effective for static networks, the mismatch between estimates and the actual environment can become significant for networks with high mobility, resulting in increased transmission failure rate. In order to achieve a better system model for networks with mobile nodes, we utilize the kinematic state of the neighbors. Along with their Vvalues, nodes include their kinematic states (position and velocity) in transmitted packet headers so that their locations can be tracked by their neighbors.

We assume that each node has some capability of estimating its own position and/or velocity. In the best case, this could be a Global Positioning System (GPS)-based system for surface nodes (buoys); submerged nodes (e.g. autonomous/unmanned underwater vehicles (AUV/UUVs)) may only have a basic inertial navigation system; fixed nodes (sensors) could be programmed with their locations at installation. For nodes that are drifting with currents and do not have such navigation capabilities, a localization algorithm could be utilized that is based on communications with neighbor nodes [18]. Note that this is a more relaxed requirement than typical of geographic routing approaches in that we do not require any node to know the location of the destination. Additionally, we do not require nodes to precisely know their locations; an uncertainty in node estimate is expected and has been modeled by normally distributed error terms applied to position/velocity measurements by the node in the simulation results presented in Section 5 .

Nodes employ a link model of the medium (either known a priori or learned) to estimate the probability of successful transmission to its neighbors based on transmission distance. For the NS-2 simulation employed, we have generated a transmission success probability model as a function of transmission power and distance using empirical equations to model sound propagation underwater. This was developed by running Monte-Carlo simulations of a closely-spaced, uniform line of nodes with a source transmitting packets at one end of the line; the number of packets successfully received at each node were counted and from this a probability of success as a function of distance was determined for various transmission power levels. The empirical equations used to model the environment account for propagation delay, attenuation due to spreading and absorption (according to the Thorp model) [19, 20], and background noise consisting of turbulence, shipping noise, surface agitation, and thermal noise [21]. Thus, it does not account for specific properties of 
any particular environment such as sound velocity profiles or bathymetry, but can be interpreted as an expected performance for a generic, uniform environment. Expected deviations of a real environment from the model have been included as normally distributed error terms.

The modeled transmission success probabilities are calculated according to:

$$
P=-\frac{s_{\text {size }}}{\pi} \arctan \frac{\left(r-r_{0.5}\right) * s_{\text {slope }}}{r_{0.5}}+v_{\text {shift }}
$$

where $s_{\text {size }}$ is an overall scaling parameter set to 1.3 and $s_{\text {slope }}$ is a scaling parameter that affects the slope of the arctangent curve, computed from a 3rd-order polynomial. The parameter $v_{\text {shift }}$ determines the vertical shift of the curve (computed from a 1st-order polynomial) and $r_{0.5}$ is the range at which the probability of successful transmission is 0.5 (computed from two different polynomials (5th order and 3rd order, respectively)). All polynomials are functions of transmission power and their coefficients were obtained by curve-fitting using the least-squares method. The resulting curves for three different transmission powers are shown in Figure 1, where we see good agreement between the model and the Monte-Carlo results.

In the case where the properties of the acoustic environment are not known a priori, these parameters could be updated using an adaptive learning scheme. This has not been implemented in this study as the primary goal of this work is to evaluate the benefit of using a model that leverages geographic information as opposed to the more simplistic method of solely using past transmission history as used by the QELAR algorithm. However, QKS also employs a method of adapting the probability based on transmission history as well: if a user-specified number of successive failures occur, the estimated probability is decreased by a fixed amount (we have set this to 0.2 ) below what is calculated from the geographic model, reflecting an assumption of bad kinematic information or model mismatch with the environment. The position estimate of the neighbor node is revised to be consistent with this new P-value estimate by moving it along the line connecting the transmitting node with the neighbor node such that the new range corresponds to that which would be expected from the probability model; the velocity estimate is reset to 0 .

Nodes maintain current estimates of their own kinematic states and also those of their neighbors using individual Kalman Filters (one for itself and for each neighbor) [22]. When packets are received/overheard from neighbors, the sender's position and velocity information is injected as a measurement to the appropriate filter. Nodes can receive a measurement update of their own position and/or velocity (depending on the node's navigation capabilities) as frequently as their 


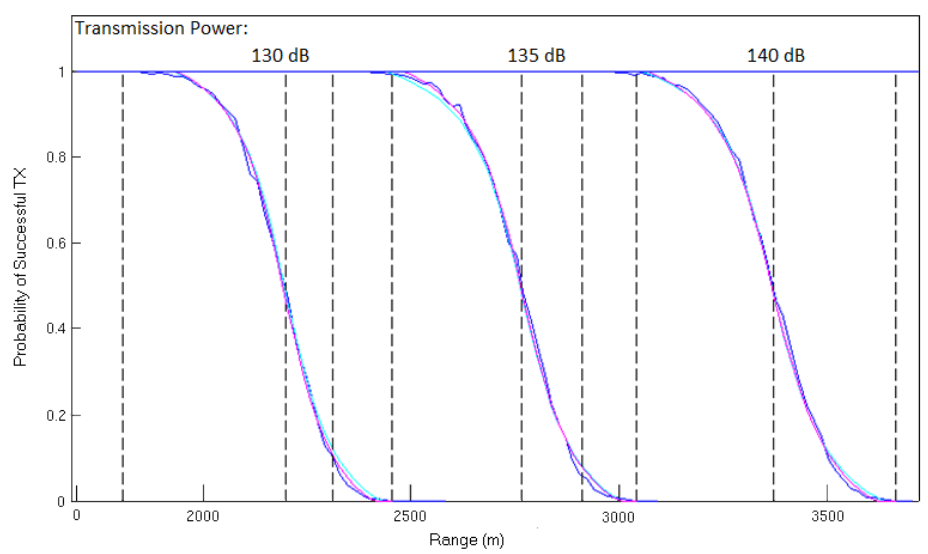

Figure 1: Transmission probability of success as a function of transmission power and distance. Each curve actually consists of three lines, nearly overlapping: Monte-Carlo simulation result (blue), custom fit curve for each transmission power (cyan), and generic model that accepts any transmission power as an input (magenta).

navigation system provides. In between measurement updates, position and velocity states are propagated forward (time updates) using a nearly constant velocity motion model.

\subsection{Selective Backward Sweeping}

As nodes learn their own V-values by communicating with neighbor nodes, there will often be significant changes in their estimated values due to initial learning or changes in the network. Under normal operation, these changes will gradually be shared as more data packets are transmitted and overheard by other nodes. Selective backward sweeping is a method of accelerating convergence by actively propagating these changes in nodes' V-values (due to learning or movement) back toward the source rather than waiting for them to percolate backward as a natural product of forwarding packets. Just as in QELAR, each time a node receives or overhears a neighbor's transmissions, it updates its own estimate of that node's V-value. However, instead of waiting until its next transmission time to compute its own V-value, it does so immediately and compares its change in $\mathrm{V}$-value, $\delta V$, to a predefined threshold, $\Theta$. If $\delta V>\Theta$, it initiates forwarding a header-only packet with its updated information so that its neighbors have access to this information.

To control the spreading of backward sweeping packets, an additional set of V-values are maintained at each node corresponding to the estimated maximum reward of going backward to reach the source node: thus, $Q^{s}\left(V^{s}\right)$ and $Q^{d}\left(V^{d}\right)$ denote the Q-values (V-values) associated with directing packets toward the source and destination, respectively. This concept of maintaining both 
forward and backward direction $\mathrm{V}$-values is similar to that described in [23], however their implementation and utilization of this information differs significantly from our approach. In [23], the $Q^{s}$-values are used in conjunction with $Q^{d}$ for routing packets to avoid bottlenecks. However, in our sweeping approach, we generate new packets containing only node header information and use the $Q^{s}$-values to route these new packets back toward the source to spread local information quickly to nodes that are not direct neighbors.

The 'selective' aspect of the approach is implemented by including a prioritized list of nodes in each packet header that indicates which ones are "allowed" to initiate backward packets. The nodes are chosen according to minimum $V^{s}$, which results in the backward packets being steered toward the source. Thus, the header of each packet now includes the node's estimate of $V_{t}^{s}$ in addition to $V_{t}^{d}$, and also a list of backward forwarder ID's. A depth parameter is included which defines how many nodes to select as backward forwarders at each transmission.

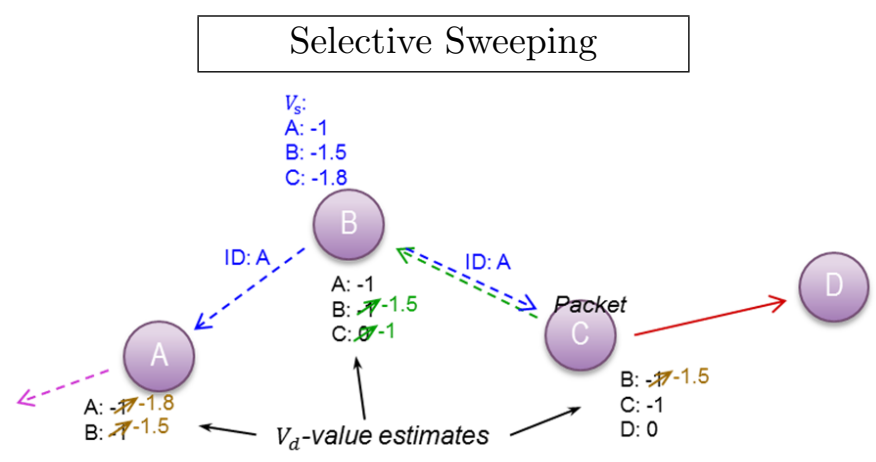

Figure 2: Five-step selective sweeping operation example. 1 (red): Node C forwards a packet to node D. 2 (green): Node B overhears the packet and updates its $V_{d}$ estimates of $\mathrm{C}$ and itself. 3 (blue): B initiates a backward packet to A since A has $\max V_{s}$ value. 4 (brown): A and $\mathrm{C}$ both hear the packet and update their $V_{d}$ estimates. 5 (magenta): Node A continues sending the backward packet since it was addressed to A.

While there is an additional overhead cost to perform the backward sweeping technique, the simulation results shown in Section 5 demonstrate that there is still an overall improvement in terms of reduced energy usage. This demonstrates that the benefit obtained in terms of improved convergence rate is effective in reducing the total number of data packet transmissions, reducing total energy usage, and therefore outweighing the cost of transmitting the sweeping packets.

This sweeping technique, and the Q-learning framework for the forward routing in QKS, has been developed and demonstrated for the single source-destination pair case. An aspect of future 
development could include generalizing this to the multiple source-destination pair case. Adding multiple sources all directing to a single destination is straightforward in regards to the normal forward routing as this still only requires a single $V^{d}$-value for each node. It would, however, complicate the selective sweeping, for which a suitable method of directing sweeping packets to one or more of the sources would need to be developed. Adding multiple destinations would require multiple $V^{d}$-values to be learned and maintained at each node. While a straightforward method of generalizing the algorithm to account for multiple source-destination pairs is easily imagined by estimating and sharing all of these additional $V^{d}$ - and $V^{s}$-values at each node and with each transmission, this also increases the overhead costs of the algorithm. It may be possible to more intelligently manage the $\mathrm{V}$-values in a joint way rather than treating them all as completely independent variables.

\subsection{Additional Features}

In addition to the main novel components just described, there are several features/modifications that were developed to provide a more robust routing algorithm.

\subsubsection{Initialization}

An initialization scheme has been developed to enable the network to automatically establish connections at the beginning of the simulation. Nodes therefore need not be initialized with any neighbor node information and can establish links to neighbor nodes autonomously. When a node has a packet to transmit but has no neighbors (e.g. when the source node produces its first packet to transmit), it places this packet on a "to_send" buffer and instead transmits a special initialization packet with an empty "next_hop" field. Each neighbor node that receives this packet can update its neighbor list to include this node as a neighbor, if it does not already know it, and also replies with an initialization packet of its own so that the original sending node will get a response and be able to establish the receiving node as a neighbor as well. In the response packet, the "next_hop" field is populated with whatever neighbors it currently recognizes. Additionally, each neighbor also adds the sending node to an "already_heard_from" list. Thus, when a node receives an initialization packet, it can detect if it has already heard from the sending node (and replied to it), or if the sending node already knows it is a neighbor (by being in the "next_hop" list) and will discard the packet in either case. This process continues until no more initialization packets are triggered due 
to these criteria - typically about one minute for the simulation scenarios presented in Section 5 . Each node has a timer which expires after 300 seconds of not receiving any initialization packets, at which time their "already_heard_from" buffers are cleared to enable the initialization process to be restarted afresh at a later time (e.g. if a node leaves and then rejoins the network).

\subsubsection{DTN Considerations}

Due to the challenges inherent in underwater communications, node mobility, and limited energy resources, underwater networks must utilize delay/disruption tolerant network (DTN) approaches to maximize network reliability. Several functionalities to address DTN issues have been developed and incorporated into the QKS algorithm.

Since nodes can become disconnected from all or a portion of their neighbors, mechanisms for reestablishing connections are required. The first way this has been implemented is that if a node overhears a packet from another node that it does not currently recognize as being a neighbor, the node will add this node to their neighbor list, and possibly also respond with a header-only packet to alert the sender that they are now neighbors so that the sender can do the same. This has been implemented with two conditions to limit the frequency of new-neighbor transmissions: the first is that the receiving node must have a $V^{d}$ estimate that is higher (better) than its estimate of the sending node, because this scenario represents a case when it would be most beneficial for the sending node to learn about this neighbor. The second condition is that the estimated probability of successful transmission must be greater than 0.5 , which insures that the extra overhead of sending a new-neighbor transmission is only used for relatively reliable links. In addition, a parameter can be set to specify the probability that a new neighbor packet would be generated even if these two criteria are met in order to further limit the number of times this happens and avoid excessive transmissions.

Another DTN feature is that if a node has a packet to transmit but does not recognize that it has any neighbors, it will first try to discover links with neighbors, and if this is unsuccessful (i.e. it is in fact disconnected from the network) it will store the packet until a future time when it reconnects with one or more nodes. The node attempts to discover neighbors by transmitting an initialization packet, which follows the procedure described in section 3.3.1.

A third feature is a time-to-live restriction which has been implemented as a hop count limit. Packets keep track of how many hops they have made and if they exceed this limit the receiving 
node will drop the packet to eliminate the possibility of packets being forwarded indefinitely (e.g. among a subset of nodes that remain disconnected from the destination).

\subsubsection{Packet Redundancy}

In both QELAR and QKS, after a node transmits a packet, it waits to overhear a future transmission of that packet from the node to which it was sent in order to know that the original transmission was successful. If the node times out before hearing such an acknowledgment, it will retransmit the packet. This works well when links are reliable, but due to the challenges of the underwater environment and network congestion, there are many times when the transmission was in fact successful, but the sending node does not overhear it due to interference or noise. In this scenario, the node will retransmit the packet, which the receiving node will then forward somewhere else, resulting in duplicate copies of that packet in the network. Duplication ensures that at least one packet will reach the destination, but also contributes to network flooding. To reduce the likelihood of this happening, a "rcvdBuffer" has been implemented at each node to record packets it has received recently; packets are cleared from the buffer after a certain time window related to the maximum round trip transmission time. If a node receives a packet that is already in its "rcvdBuffer" (matching packet ID, previous hop node, and hop count) it knows that this is a duplicate packet and will respond with a header-only acknowledgment packet to let the sender know it was received. Unnecessary duplicate packets can still arise from various other situations, which could be addressed in other ways (see Section 6).

\section{Baseline Flooding Algorithm}

In addition to the reinforcement learning algorithms focused on in this work, a simple floodingbased algorithm called Information Carrying Based Routing Protocol (ICRP) has been simulated as well to produce a baseline routing comparison with a non-RL approach [12].

The source node initiates route establishment by broadcasting a packet to all of its neighbor nodes. Each of these nodes in turn broadcasts the packet, while maintaining a record of the route the packet took. When the destination receives the packet, it sends a "status" packet back to the source node along the reverse route, thereby establishing a route between the source and the destination. This route is used until it is disrupted (perhaps due to node mobility), at which point packets are again broadcasted to find a new route. 
For the purposes of comparison with the QELAR and QKS algorithms, similar metrics are computed for the simulations shown in the next section. While most of the metrics are directly comparable (total energy used, total number of transmissions, etc.) there are two that do not directly translate and are computed differently for ICRP: total failed transmissions and total periodic transmissions. The total number of failed transmissions can only be computed for those packets that are directed to a specific next neighbor, not broadcasted packets, as these are the only packets that require an acknowledge from the receiving node to determine success/failure. Therefore the recorded number of failures is lower than the other algorithms. The second metric, total periodic transmissions, is redefined to count the status packets that are sent to establish new routes.

\section{Simulation Results}

The popular NS-2 network simulator was used to implement both the baseline and modified algorithms [16]. Several extensions have been leveraged to make use of existing underwater modeling (DESERT [24] and WOSS [25]) as well as NS-MIRACLE [26] to support modularization of the code.

While multiple sources/destinations could exist in the simulator, the results presented here include a single source and single destination. The source node generates packets using the constant bit-rate (CBR) module provided in the DESERT framework which produces packets at a fixed period, which is scenario specific, and bit rate of $4800 \mathrm{bit} / \mathrm{s}$. Packets consist of a header and a payload. Header fields depend on the algorithm implemented (see Table 1) and serve to direct the packet along its path and relay routing information among nodes. The size of the header is estimated by allowing one byte for each field that is a node ID (enabling 256 nodes to be present in the network - this could be expanded if necessary) and two bytes for each numerical value. The payload section contains the actual data being transmitted by the source and is fixed at 200 bytes for all packets.

An energy module has been developed to record energy usage at each node. It models the transmission, receiving, and idle power levels to be $10 \mathrm{~W}, 3 \mathrm{~W}$, and $0.03 \mathrm{~W}$, respectively, and deducts energy from an initial available amount (e.g. $20 \mathrm{~kJ}$ ) as nodes spend periods of time in each of these states. The simulation continues for a specified period of time, 7200 seconds (2 hours) in the cases presented here. 
Table 1: Packet header fields for QELAR (Q), QKS with kinematic tracking only (T), and QKS with backward sweeping only (S). Note that 'Next Backward ID' is a vector of ID's, with length depending on the sweep depth used; 3 bytes are listed as a representative size.

\begin{tabular}{|c|c||c|c|c|}
\hline Field & Bytes & Q & T & S \\
\hline \hline Packet ID & 1 & $\mathrm{x}$ & $\mathrm{x}$ & $\mathrm{x}$ \\
\hline Dest Node ID & 1 & $\mathrm{x}$ & $\mathrm{x}$ & $\mathrm{x}$ \\
\hline Sender $V_{d}$ & 2 & $\mathrm{x}$ & $\mathrm{x}$ & $\mathrm{x}$ \\
\hline Energy (res) & 2 & $\mathrm{x}$ & $\mathrm{x}$ & $\mathrm{x}$ \\
\hline Energy (avg) & 2 & $\mathrm{x}$ & $\mathrm{x}$ & $\mathrm{x}$ \\
\hline Prev Hop Node ID & 1 & $\mathrm{x}$ & $\mathrm{x}$ & $\mathrm{x}$ \\
\hline Next Forwarder ID & 1 & $\mathrm{x}$ & $\mathrm{x}$ & $\mathrm{x}$ \\
\hline Sender Pos & 6 & & $\mathrm{x}$ & \\
\hline Sender Vel & 6 & & $\mathrm{x}$ & \\
\hline Sender $V_{s}$ & 2 & & & $\mathrm{x}$ \\
\hline Next Backward ID & 3 & & & $\mathrm{x}$ \\
\hline
\end{tabular}

Results from two different scenarios are shown: a fixed grid with mobile UUV, and a random field of nodes with different levels of mobility. A Monte-Carlo simulation mode has been employed, where identical initial node configurations are used, but the random aspects of the simulation will vary (e.g. node movement, packet errors due to noise, etc.). Each algorithm is run between 15 and 50 times, depending on the variability/mobility of the scenario, limited by computational time. The results are averaged across all runs to provide a more representative result, especially for different configurations that can occur in mobile scenarios.

Various algorithm configurations have been run for comparison. The QELAR baseline algorithm has been evaluated both using time-based periodic transmissions, and without, to show the effect of the transmissions. The period of the periodic transmissions has been fixed to 1000 seconds: several values were tested and this was chosen as providing good results for various configurations. Six configurations of the QKS algorithm have also been run: tracking only, selective sweeping with depths of 1,3 , and 5, uniform sweeping, and periodic (incorporating the time-based periodic transmissions used by QELAR instead of the selective sweeping technique). In "uniform" sweeping, instead of selectively choosing nodes with the minimum $\mathrm{V}$-values in the direction of the source node, all neighbors are allowed to transmit sweeping packets. This is therefore the upper limit on the amount of sweeping that can be performed. Results using a simple flooding-based routing protocol 
(ICRP) are also shown for comparison. Parameters used for all algorithm configurations and scenarios are listed in Table 2.

A number of metrics have been computed to compare algorithm performance under both static and mobile scenarios (Tables 3 and 5). 'Total Energy' is the sum of all energy consumed by all nodes during the simulation. 'Total TX', 'Failed TX', and 'Total RX' are the total transmissions of all nodes, the number of transmissions that failed to reach their intended next hop, and the total number of successful packet receives at all nodes, respectively. Note that for the ICRP algorithm, the 'Failed TX' accounts only for those packets that were forwarded to a particular node, not any broadcast packets, and thus is significantly lower than the reinforcement learning algorithms where all packets are forwarded to specific nodes. 'Sweep TX' denotes the number of transmissions initiated as part of the selective sweeping functionality (QKS) and 'Total Periodic TX' is the total number of periodic transmissions (consisting of time-based and those triggered by new neighbors ('New Neighbor Per') for QELAR and QKS, and status packets for ICRP). 'Avg Path Len' is the average path length considering all paths from source to destination used by packets during the simulation. 'Avg Latency' is the average time, in seconds, between a packet being transmitted from the source and first reaching the destination node.

Table 2: Parameter values used in all simulations.

\begin{tabular}{|c|c|l|}
\hline Parameter & Value & Function \\
\hline \hline$g$ & 1 & Constant transmission cost \\
\hline$\alpha_{1}$ & 0.1 & Weight of $c(s)$ reward term for successful transmission \\
\hline$\alpha_{2}$ & 0.01 & Weight of $d(s)$ reward term for successful transmission \\
\hline$\beta_{1}$ & 0.1 & Weight of $c(s)$ reward term for failed transmission \\
\hline$\beta_{2}$ & 0.01 & Weight of $d(s)$ reward term for failed transmission \\
\hline$\gamma$ & 0.8 & Discount parameter to control short- vs. long-term benefit \\
\hline$\eta$ & 2 & Additional weight factor affecting cost of transmission for failure case \\
\hline$\delta V$ & 0.4 & Change in V-value threshold for selective sweeping (QKS only) \\
\hline TTL & $50 ; 10$ & Time-to-live (number of hops) for each packet (QELAR/QKS;ICRP) \\
\hline
\end{tabular}

It has been assumed that nodes are able to estimate their own position but are unable to directly measure their own velocity - all velocity calculations are derived from the Kalman filter state as a result of the position measurements. Node position measurements have normally distributed errors with a variance of $50 \mathrm{~m}$, resulting in a mean position error of approximately $11 \mathrm{~m}$. The normally 
distributed errors applied to the probability of success model used by the QKS algorithm were generated with a standard deviation of 0.05 .

\subsection{UUV Scenario}

The first scenario consists of a fixed grid of stationary nodes with $1 \mathrm{~km}$ spacing and one mobile node which serves as the destination (see Figure 3). This can be imagined as a grid of fixed sensors/relay nodes with a UUV traversing the grid, which one sensor is trying to transmit data to, using the remaining nodes as relays as necessary. The path of the mobile node (26) is shown in blue; node 1 is the source node for the entire simulation. The source packet generation period has been set to 60 seconds for this scenario. ${ }^{1}$

Table 3 shows the metrics calculated for this scenario for all algorithm configurations run. Some key metrics are also shown graphically in Figure 4. Similar performance is observed between the ICRP and QELAR algorithms in terms of energy usage and total transmissions, but ICRP suffers reduced packet delivery to the destination due to difficulty in establishing a route when the UUV is at the far side of the field from the source. There is approximately a factor of two reduction in total energy usage by all of the QKS implementations as compared to QELAR, primarily due to the fact that the tracking component of QKS allows the nodes to converge toward their actual V-values more quickly and also track, and therefore adapt to, mobility much better. Additionally, as a result of the improved convergence and tracking, we see even more significant reductions in failed transmissions for QKS over QELAR. Due to the organized structure of the field, transmission power can be scaled to achieve reliable connections between adjacent nodes, but minimal interference to more distant ones, resulting in a relatively easy scenario (good connectivity with minimal congestion) and 100 percent packet delivery success to the destination for both RL-based algorithm implementations.

It is noted that latency is much lower for the QKS implementations over QELAR, but that ICRP also has a low latency. This is due to the fact that ICRP also has a lower success rate, and packets that do not reach the destination at all are not factored into the latency calculations.

\footnotetext{
${ }^{1}$ To evaluate the performance under heavier network loads, this scenario was also run with a packet generation period decreased to 30 seconds. Results are not shown due to space, but the QKS algorithm was able to maintain similar performance, with metrics scaling as expected (energy and total transmissions increase by slightly less than a factor of two). Delivery success remains at $100 \%$. The QELAR algorithm, however, suffers in performance with total energy usage increasing by a factor of five and packet delivery to the destination decreasing to around $80 \%$.
} 


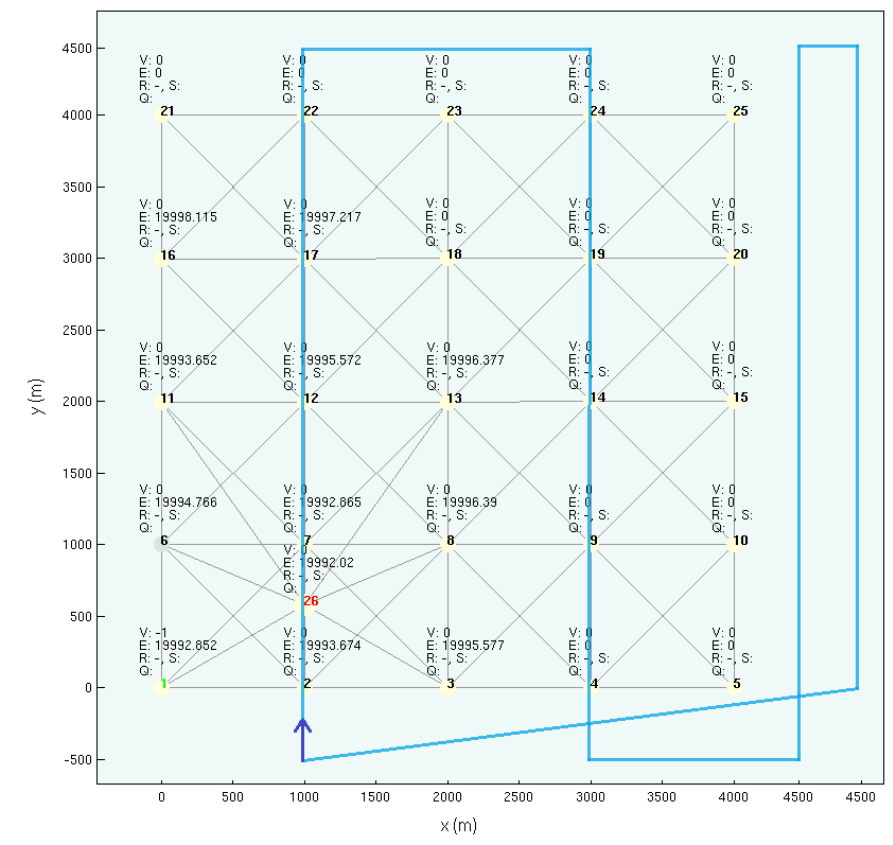

Figure 3: Field layout; UUV starts at $(1000,-500)$ and follows the path shown in blue, shown here at position $(1000,550)$ part way into run. The source is indicated by the node numbered in green (node 1 ) and the destination is numbered in red (node 26 - UUV). Gray lines indicate node connections with a probability of success greater than 0.5 .

That is, ICRP achieves a low packet latency at the expense of delivery reliability, whereas QKS with sweeping is able to achieve both. Maximum latencies for ICRP, QELAR, and QKS algorithm implementations are approximately 20,600, and 150 seconds, respectively, with all implementations having identical minimum latencies of 1.5 seconds, corresponding to direct transmission from source to destination at the beginning of the scenario.

Comparing the six different QKS implementations, we can observe the further improvement gained by augmenting the tracking capability with the selective sweeping strategy. For this scenario, the sweeping level of 5 produces the best results over all of the metrics calculated - other levels may produce better results in other scenarios, as will be seen in Section 5.2. Results shown for QKS when using time-based periodic transmissions, as implemented in QELAR, instead of selective sweeping is also shown to demonstrate the shortfalls for this approach in certain situations. While there is a slight reduction in total energy usage as compared to the QKS implementation with tracking only, it is not as significant as that obtained with the sweeping; the reason can be seen by looking at the transmission counts: there are more transmissions triggered and the number of failures is 
Table 3: Metrics for UUV scenario. Algorithms implemented are flooding (ICRP); QELAR, with and without timebased periodic transmissions; and QKS using tracking (T), selective sweeping (S) with various depths: 1neighbor $(\mathrm{S}(1)), 3$ neighbors $(\mathrm{S}(3))$, 5 neighbors $(\mathrm{S}(5))$, or uniform $(\mathrm{S}(\mathrm{U}))$, and time-based periodic transmissions. *Failed TX and Total Periodic TX have unique definitions for ICRP case (see Section 4 text).

\begin{tabular}{c|c|cc|cccccc}
\hline Algorithm & \multirow{2}{*}{ ICRP } & \multicolumn{2}{|c|}{ QELAR } & \multicolumn{5}{c}{ QKS } \\
& & no P & P & T Only & T\&S(1) & T\&S(3) & T\&S(5) & T\&S(U) & T\&P \\
\hline \hline Total Energy (J) & 28007 & 28300 & 26600 & 14700 & 13600 & 13200 & 12800 & 12900 & 14400 \\
\hline Total TX & 2518 & 2018 & 1920 & 836 & 787 & 770 & 742 & 768 & 877 \\
\hline Failed TX & $10^{*}$ & 1033 & 903 & 119 & 69 & 54 & 39 & 48 & 76 \\
\hline Total RX & 14743 & 12419 & 12500 & 5850 & 5470 & 5360 & 5130 & 5300 & 6200 \\
\hline Sweep TX & 0 & 0 & 0 & 0 & 40 & 77 & 80 & 103 & 0 \\
\hline Total Periodic TX & $240^{*}$ & 1 & 118 & 0.5 & 0.8 & 0.2 & 0.5 & 0 & 102 \\
\hline New Neighbor Per & 0 & 1 & 7.2 & 0.5 & 0.8 & 0.2 & 0.5 & 0 & 7.8 \\
\hline Avg Path Len & 13 & 15 & 13 & 5.3 & 4.6 & 4.1 & 3.9 & 3.9 & 4.8 \\
\hline Avg Latency (s) & 9 & 120 & 78.8 & 14.4 & 11.4 & 9.6 & 8.7 & 8.9 & 11.9 \\
\hline Percent Delivered & 82.3 & 99.7 & 100 & 100 & 100 & 100 & 100 & 100 & 100 \\
\hline
\end{tabular}

not as reduced as with selective sweeping. This is due to the fact that the selective sweeping technique utilizes the nodes' convergence estimates in generating packets and thus generates many packets when they are valuable but does not generate them when they are unnecessary, whereas the time-based periodic do not adapt to the network convergence.

Figures 5 and 6 provide insights into how well the nodes of the network converge in $\mathrm{V}$-value estimate, and adapt to the mobility of the UUV. The actual V-values for each node are computed at each time instant by iterating the Q-learning equation (7) at all nodes in the network until they converge, and each node's estimated V-value is compared to the correct value; the deviations between these two are averaged for all nodes. This provides an overall metric of how well the network as a whole has converged at any given time; note that zero-error convergence can never happen because the network is attempting to converge on a "moving target" since the V-values are constantly changing. It should also be noted that some level of overall error in $\mathrm{V}$-value estimate in the network is not necessarily a bad thing, because if nodes are not located along a path used to get to the destination, it may be acceptable to let their $\mathrm{V}$ estimates be inaccurate and not waste resources updating them. However, this metric still gives valuable insight into routing performance.

All nodes are initially set to have a $\mathrm{V}$-value of 0 . In actuality, the only node whose correct 


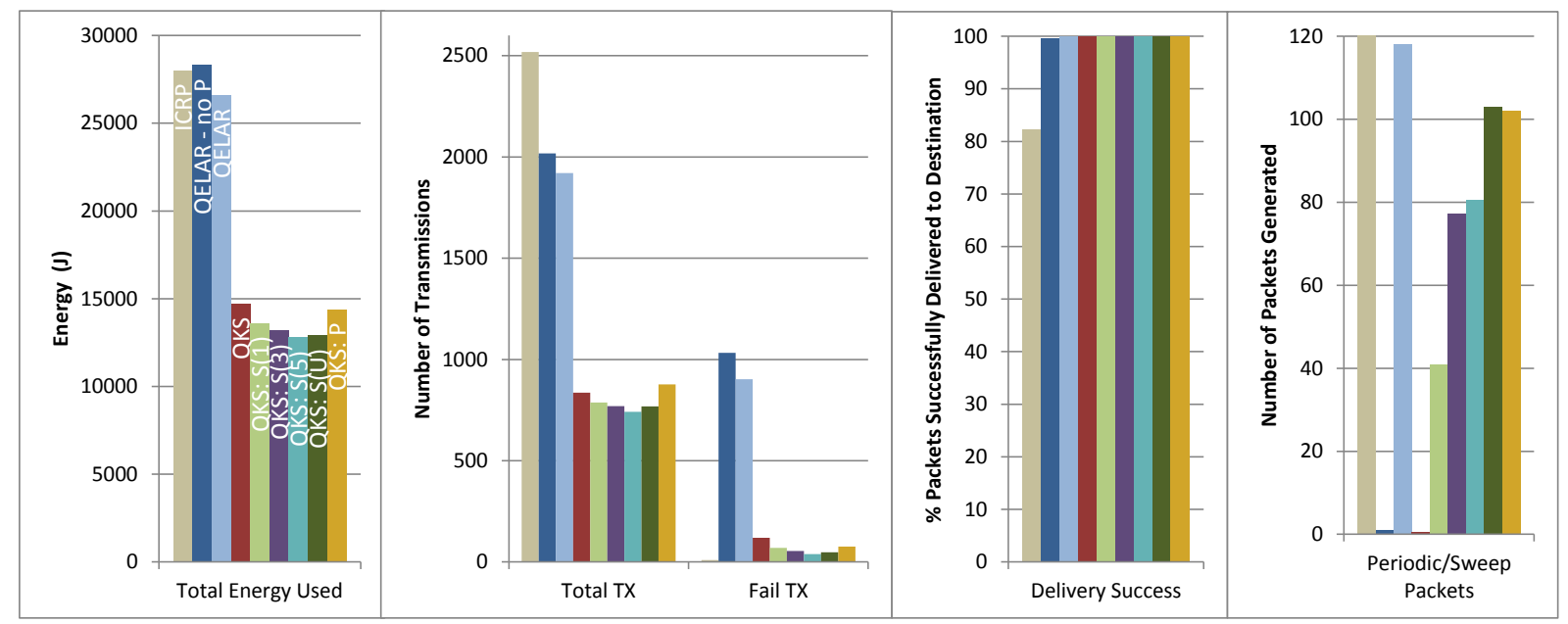

Figure 4: Graphical representation of selected metrics for the mobile UUV case. Algorithms compared (shown in same order on each graph): ICRP, QELAR (without/with periodic TX), QKS (tracking only), QKS (sweeping depths of $1,3,5$, and uniform), and QKS with periodic TX only.

value is 0 is the destination node. As the network learns, all other nodes decrease their $\mathrm{V}$-values (all other $\mathrm{V}$ are negative) indicating a measure of how far they are from the destination. Ignoring energy considerations (i.e. setting $\alpha$ and $\beta$ in equations (4) and (5) to 0), nodes that are immediate neighbors of the destination have a $\mathrm{V}$-value of -1 . With $\gamma=0.8$, as is the case for these simulations, nodes that are two hops away would have V-values of -1.8 ; three hops -2.44 ; four hops -2.95 ; and so forth, converging to -5 as nodes become an infinite number of hops from the destination. Accounting for energy makes these numbers slightly lower (more negative), but a similar trend and scale of values still holds.

The average V-value error for all nodes (Figure 5) is an estimate of how well the network as a whole is able to discover and track changes in V-values as the configuration changes due to the UUV movement. All RL algorithm configurations are shown, but special attention can be drawn to the bold lines, which show the performance of the QKS algorithm with no sweeping or periodic transmissions, which corresponds to the worst V errors (of the QKS implementations), and the QKS with uniform selective sweeping, which corresponds to the minimal V errors. QELAR demonstrates the worst errors overall. In the first 1000 seconds, QELAR actually shows lower error than some of the QKS algorithms due to the fact that it does not quickly identify efficient routes to the destination at the beginning, and therefore routes packets unnecessarily throughout the network. The result is that all of the nodes begin updating their $\mathrm{V}$-values from the beginning of the scenario, 


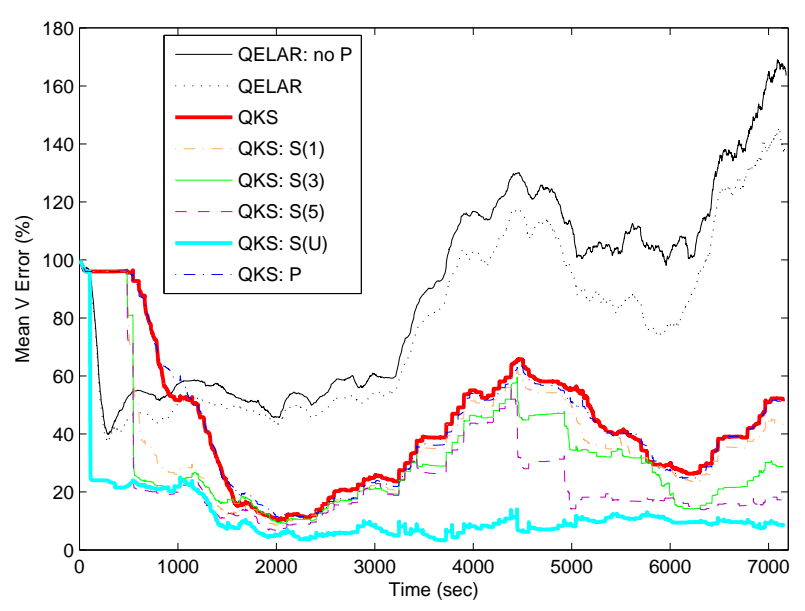

Figure 5: Mean V-value estimate error for all nodes over time for each algorithm configuration.

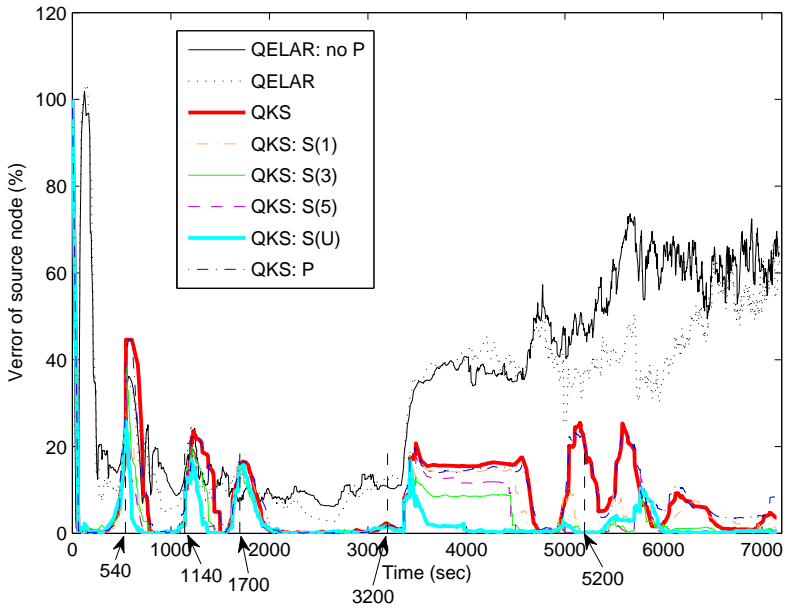

Figure 6: V-value estimate error of source node over time for each algorithm configuration.

whereas with QKS, packets are routed more directly to the destination and thus nodes that are more distant do not start to update until later. However, QKS with selective sweeping increases the convergence rate of all nodes in the network due to the spread of the selective sweeping packets.

It is also valuable to consider the $\mathrm{V}$ error of just the source node, as this should always be as low as possible and is a reflection of the convergence of the key nodes on the path between the source and destination. As Figure 6 shows, the 'QKS: $\mathrm{S}(\mathrm{U})$ ' implementation shows the best performance, with the 'QKS' (tracking only) demonstrating more limited ability to track the $\mathrm{V}$ error but still out-performing QELAR. A summary of key events that correspond to artifacts in the plot are listed in Table 4. The benefit of sweeping can most clearly be seen by looking at the period from 3500 to 4500 seconds. This corresponds to the time when the UUV re-enters the field at the top and heads down over nodes 24,19 , etc. Prior to this time, these nodes had lower V-values because they were far from the destination, but now they are suddenly immediate neighbors and update their $\mathrm{V}$-values accordingly. When using selective sweeping, this triggers sweeping packets to be generated which propagate this information back toward the source, and thus we see that the versions of the algorithm with sweeping reduce the $\mathrm{V}$ error of the source more quickly in this period than the algorithm without.

To further understand the reason for the differences in performance between the QELAR and QKS algorithms, we can look at how well the nodes are able to estimate the probability of success to their neighbors. Figure 7 shows the average total error in P-value estimates for the entire network 
Table 4: Description of noteworthy events in Figure 6.

\begin{tabular}{|c|c|c|}
\hline Time & UUV Location & Event Characterization \\
\hline 540 & $\begin{array}{l}\text { Heading up on first leg, near } \\
\text { node } 12\end{array}$ & $\begin{array}{l}\text { Source can no longer transmit directly to destination - starts using } \\
2,6 \text {, and } 7 \text { as relay nodes; two hops required to reach destination; } \\
\text { source error spike due to suddenly not being neighbor of destination }\end{array}$ \\
\hline 1140 & $\begin{array}{l}\text { Heading up on first leg, near } \\
\text { node } 17\end{array}$ & $\begin{array}{l}\text { Packets must now make three hops to the destination; begin ex- } \\
\text { ploring the bottom right quadrant of the field }\end{array}$ \\
\hline 1700 & $\begin{array}{l}\text { Heading up on first leg, near } \\
\text { top of field }\end{array}$ & Packets must now make four hops to reach destination \\
\hline 1900 & UUV leaves field (top) & \\
\hline 3200 & $\begin{array}{l}\text { UUV re-enters field near } \\
\text { node } 24\end{array}$ & $\begin{array}{l}\text { Nodes near } 24 \text { now immediate neighbors of UUV; update their V- } \\
\text { values accordingly; source learns of this with some lag (reduced } \\
\text { when using sweeping) }\end{array}$ \\
\hline 5200 & UUV leaves field (bottom) & \\
\hline 6300 & $\begin{array}{l}\text { UUV begins upward leg on } \\
\text { right side of field }\end{array}$ & \\
\hline
\end{tabular}

as a function of time for each algorithm. That is, for each node, the P-value estimates to all other nodes in the network are summed at each time instant and compared to the actual (modeled) P-values using true node locations. These errors are then averaged across all nodes to produce the curves shown in the plot. There is significant improvement by any of the QKS algorithms over QELAR due to the fact that nodes use geographic information to update their $\mathrm{P}$ estimates rather than just a history of successes/fails. There is also slight improvement shown by the inclusion of selective sweeping or periodic transmissions. The implementation of QKS with time-based periodic transmissions actually outperforms the selective sweeping at some points due to the fact that it will trigger nodes that are not on the path from source to destination to periodically update based on time, whereas the selective sweeping will allow these nodes to remain inaccurate in $\mathrm{P}$-value estimate since it is not necessary to update them.

\subsection{Random Node Scenario}

The second scenario consists of 50 randomly placed nodes within a volume of $1500 \times 1500 \mathrm{x}$ $1000 \mathrm{~m}^{3}$. The scenario was run in six different configurations: all nodes being static, four different fractions of nodes being mobile $(5,10,20,30)$, and all nodes being mobile. Mobile nodes are initialized with a random direction and random speed (mean speed of $1 \mathrm{~m} / \mathrm{s}$ ); they follow straight 


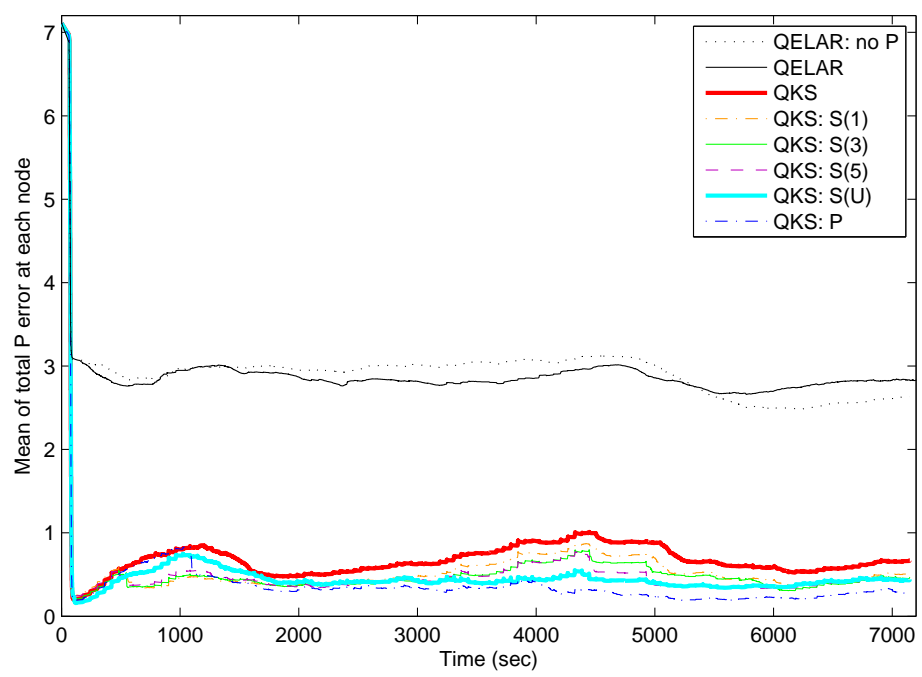

Figure 7: Average total error in probability of successful transmission of each node to its neighbors for the entire network.

line paths and reflect off of the boundaries of the bounding area if they reach it. Nodes are modeled as having an ability to estimate their own position (with some uncertainty), but not their velocity; velocities are calculated using the Kalman Filter state estimates only.

The packet generation at the source node has been slowed down to have a period of 150 seconds. This is much slower than the network could handle once it has converged, but the initial packets take very circuitous routes to the destination, and thus a faster packet rate can tend to flood the network during this learning phase. An adaptive rate would be a logical choice but has not yet been implemented. However, a carrier sense medium access control (MAC) protocol has been implemented which provides a limited level of interference mitigation.

Similar to the UUV case, we again see significant reductions in total energy usage for the QKS algorithm as compared to QELAR due to reduced total and failed transmissions (see Table 5). While in the first scenario this was primarily due to QELAR's inability to quickly adapt to changes in the network, it occurs in this static case because of the large search space at the beginning of the scenario. QELAR inherently requires multiple transmission attempts to estimate P-values, and thus it takes an appreciable amount of time for the estimates to be accurate. During this time, routing decisions may be poor, resulting in failed transmissions, duplications of the packet, and network congestion. However, QKS gets an instantly accurate (within the accuracy of the position information) P-value estimate after only one transmission, resulting in better forwarding choices, 


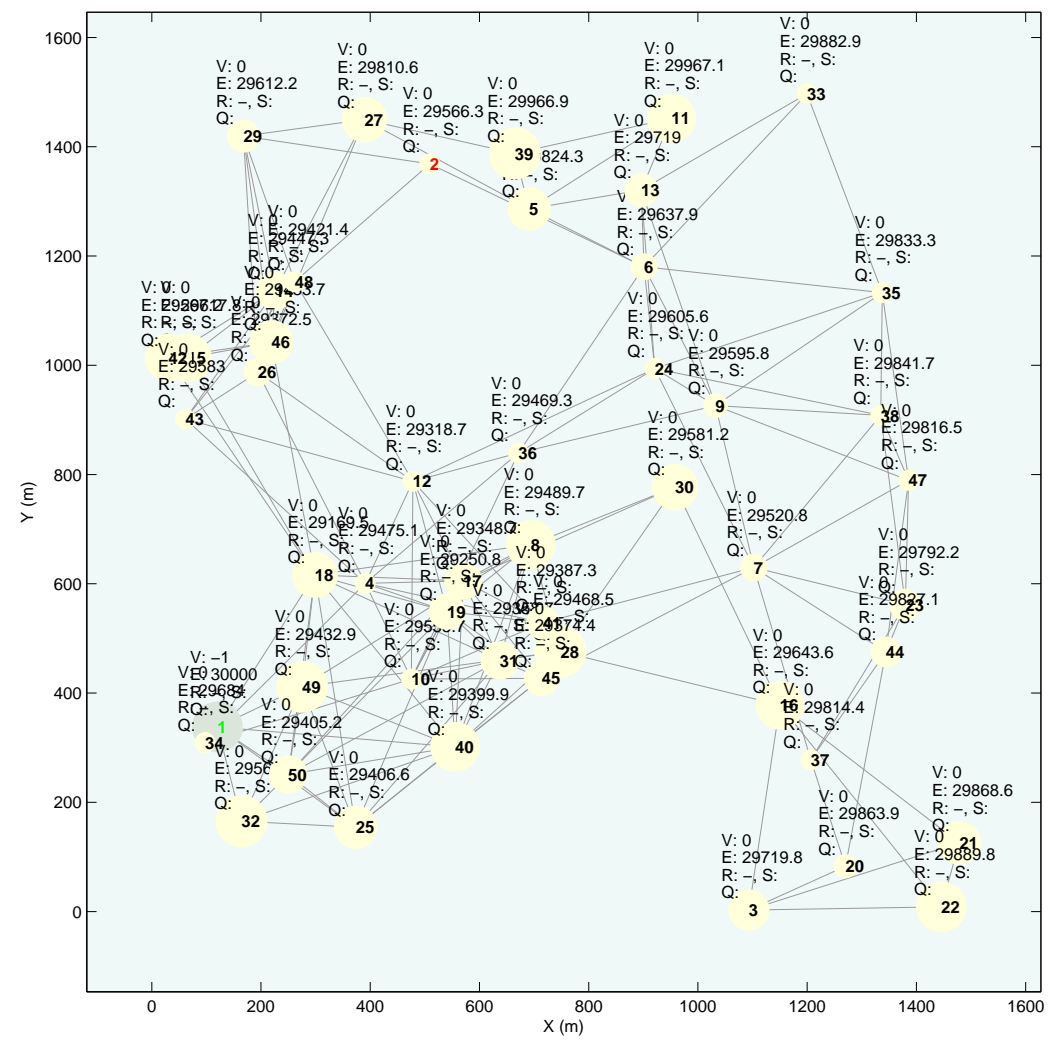

Figure 8: The (initial) random layout for both static and mobile cases; the source node (1) is labeled in green and the destination (2) is labeled in red. The node size indicates depth, with deeper nodes appearing smaller.

especially for the initial packets of the scenario. In addition to increased energy usage, this challenge to converge for QELAR also results in some packets not being able to reach the destination at all due to network flooding, and thus there is only about a $50 \%$ success rate on average, while QKS remains near $100 \%$. However, the ICRP algorithm fails to deliver many packets to the destination, and in fact, rarely is able to establish a route to the destination node at all due to congestion and packet interference from the flooding packets due to the density of the nodes in this scenario. Total energy usage is low, near that of QKS, due to the fact that packets are dropped after 10 hops due to the time-to-live restriction. The latency of IRCP is unable to be computed due to the low delivery success rate, but that of QELAR is significantly higher than QKS and corresponds with the increase in average path length.

Some nodes were then specified to move to examine algorithm performance in the presence of mobility. First, 5 nodes were specified to be mobile while the remaining 45 were stationary. Then 10 were made mobile with 40 stationary, and similarly for 20 and 30 mobile nodes. Finally, all 
Table 5: Metrics for 50 static, random node scenario.

\begin{tabular}{c|c|cc|cccccc}
\hline \multirow{2}{*}{ Algorithm } & \multirow{2}{*}{ ICRP } & \multicolumn{2}{|c}{ QELAR } & \multicolumn{5}{c}{ QKS } \\
& & no P & $\mathrm{P}$ & T Only & T\&S(1) & T\&S(3) & T\&S(5) & T\&S(U) & T\&P \\
\hline \hline Total Energy (J) & 28452 & 104000 & 94100 & 22200 & 21300 & 20800 & 20400 & 22100 & 24300 \\
\hline Total TX & 1289 & 8040 & 6650 & 987 & 920 & 958 & 948 & 1056 & 1177 \\
\hline Failed TX & 0.4 & 5790 & 4140 & 293 & 238 & 231 & 216 & 240 & 256 \\
\hline Total RX & 1957 & 19500 & 21300 & 6230 & 5930 & 6160 & 6000 & 6283 & 7817 \\
\hline Sweep TX & 0 & 0 & 0 & 0 & 8.3 & 95 & 105 & 152 & 0 \\
\hline Total Periodic TX & 4 & 15 & 196 & 6.3 & 6.3 & 4.8 & 2.5 & 0 & 245 \\
\hline New Neighbor Per & 0 & 15 & 16 & 6.3 & 6.3 & 4.8 & 2.5 & 0 & 9.5 \\
\hline Avg Path Len & 27.1 & 159 & 126 & 15.5 & 14.1 & 13 & 12.5 & 13.1 & 14.6 \\
\hline Avg Latency (s) & N/A & 563 & 673 & 57.4 & 83.9 & 51.2 & 43.3 & 46.8 & 51.1 \\
\hline Percent Delivered & 4.5 & 57.2 & 48.1 & 98.3 & 97.4 & 99.8 & 99.6 & 100 & 98.7 \\
\hline
\end{tabular}

of the nodes were made mobile. Total energy and percent of packets successfully delivered to the destination are plotted in Figure 9 for all algorithm implementations as a function of the number of mobile nodes. The ICRP algorithm was not compared for the mobile case due to the poor delivery performance even for the static scenario.

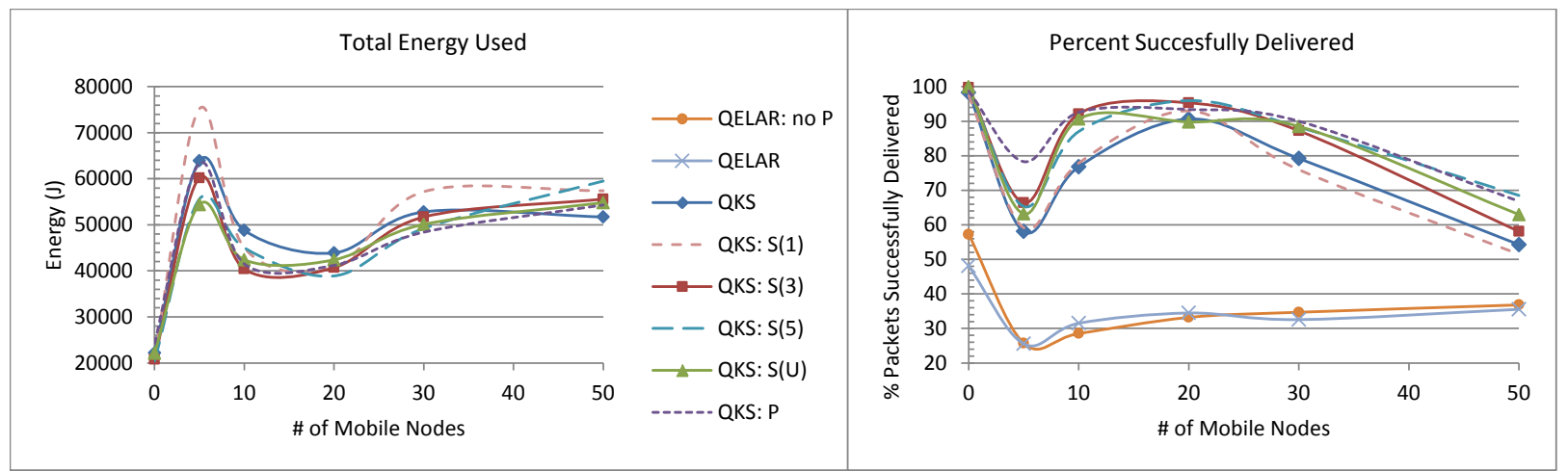

Figure 9: Total energy used (left) and percent of packets successfully delivered to the destination (right) for all algorithm implementations as functions of number of mobile nodes. Note that the ordinate axis of the energy plot has been scaled to exclude the QELAR results (which are in excess of 100,000 J) to provide greater clarity to QKS curves.

It was expected that a steady progression would be found of increasing energy usage and reduced successful packet delivery as more nodes became mobile. However, we see an intermediate peak (poor performance) at the five mobile node case, and an opposite peak (good performance) at the 
20 mobile node case. This is due to a unique characteristic of the field layout. In the initial node layout, there are three nodes that can transmit to the destination: 6, 29, and 48; the closest and most reliable is node 48. However, node 48 is one of the first five nodes to become mobile, and therefore, this link is broken and packets are forced to use the other, less reliable, links to reach the destination node. As mobility increases further, other nodes periodically fill in these gaps, resulting in overall improved performance. However, at a certain point (greater than 20 mobile nodes) the challenges of tracking the high mobility starts to dominate and performance falls off again.

Note that the vertical axis of the energy plot has been limited to the QKS results - the QELAR results would be significantly higher, and are not particularly meaningful due to the poor performance of QELAR in the presence of mobility. The QELAR results shown in the packet delivery plot, however, show similar poorer performance in the 5 mobile node case, but instead of rebounding to a maximum near 20, it remains relatively constant. This indicates that QELAR is unable to track the mobility, as QKS is able to do, and thus cannot take advantage of the nodes moving near the destination, unlike QKS.

The V-value estimate error of the source node is plotted for the scenario with 20 mobile nodes in Figure 10. Due to the random motion and the Monte Carlo averages, the curves are less erratic than we saw in source node error of the UUV case. We once again see similar trends in terms of the QKS algorithm, with the implementation with no sweeping or periodic packets producing the worst result and QKS with sweeping of depth 5 or uniform producing the lowest error and fastest convergence. The QELAR implementations are significantly worse, especially in terms of growing error as time progresses.

The key metrics are shown for the 20 mobile node case in Figure 11. The vertical scale has been chosen to focus on the QKS results - the QELAR bars actually extend significantly above the chart, as evidenced by the metrics shown in the static case (Table 5). We still observe noticeable improvement by the selective sweeping technique, but it is less significant than we observed in the mobile UUV case due to the more challenging characteristics of the random movement. We also observe that QKS with time-based periodic transmissions produces results that are more similar to that of sweeping than we saw previously. This is because as mobility increases, nodes have a harder time maintaining accurate $\mathrm{V}$-value estimates. This affects the ability of the selective sweeping paradigm to intelligently trigger packets to be generated, whereas the periodic approach 


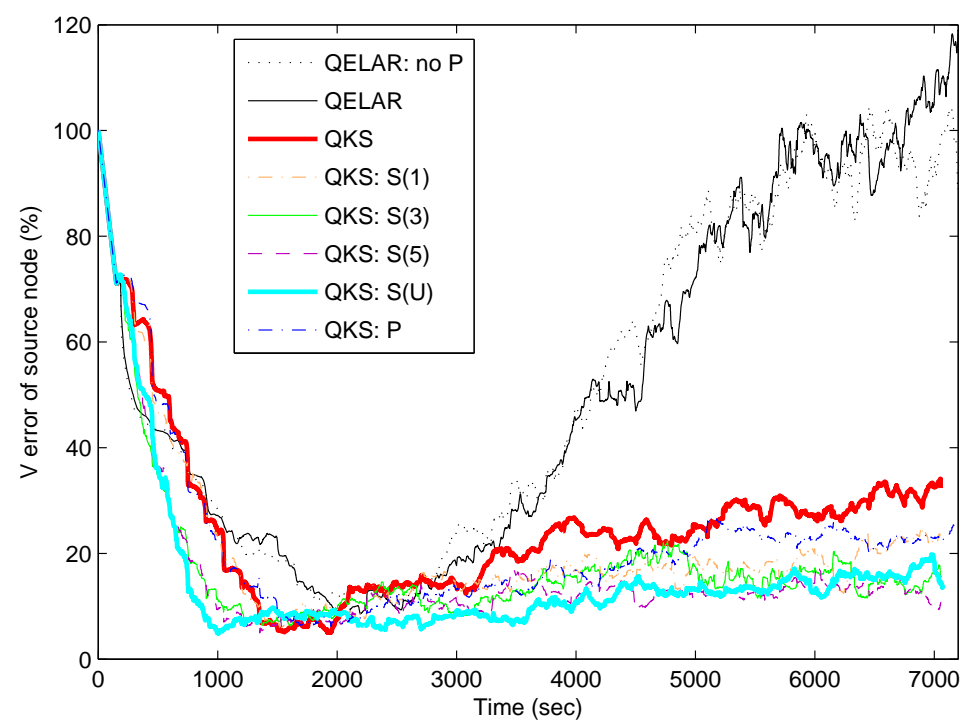

Figure 10: Source node V-value estimate error for all algorithm implementations for the 20 mobile node (30 static node) scenario.

is not affected by how well the network is able to converge. However, the selective sweeping is still advantageous in that it adapts to the changes in the network as opposed to the periodic transmission approach; i.e., if the mobile nodes became stationary in the middle of the scenario, the selective sweeping would reduce in frequency to save energy and reduce congestion as seen in the static case.

\section{Conclusion and Future Work}

Our RL-based routing algorithm employing the features of tracking using neighbor node kinematic information and prioritized sweeping based on changes in V-value estimates demonstrates faster convergence and better estimates of dynamic networks than the baseline algorithm, QELAR. This results in decreased overall energy usage, which is a key limitation in underwater networks.

There are several modifications that we plan to incorporate into the algorithm that should further improve the performance and throughput of the network. We envision that the packet generation at the source node should adapt to the network status (convergence, packet traffic, etc.) by increasing the packet generation rate and/or packet size when it estimates that the network has converged (the nodes along the path from itself to the destination accurately know their neighbor links and V-values). Additionally, all nodes should adjust their transmission power on a per-hop 


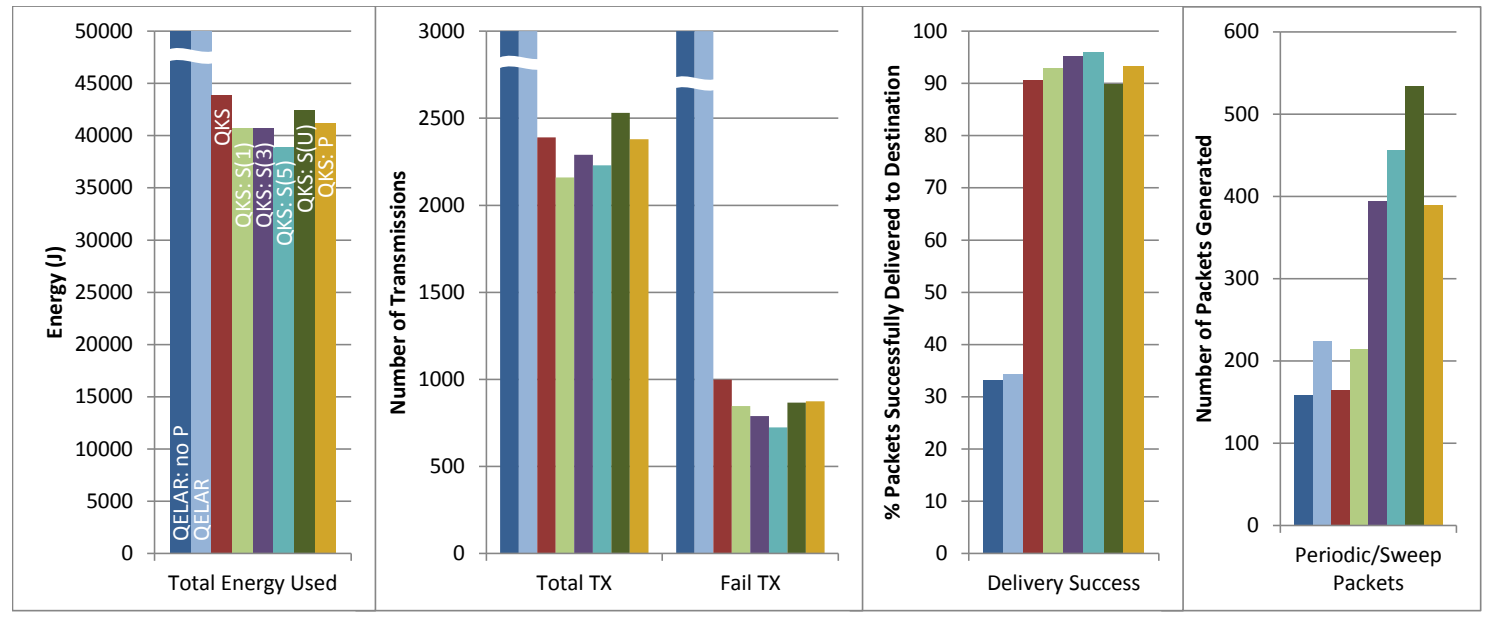

Figure 11: Selected metrics for 20 mobile node scenario. Algorithms compared (shown in same order on each graph): QELAR (without/with periodic TX), QKS (tracking only), QKS (sweeping depths of 1, 3, 5, and uniform), and QKS with periodic TX only. Note that the vertical axes of the energy and transmission count plots have been zoomed to the QKS results - QELAR bars extend above maximum of chart.

basis, such that less power is used when the desired neighbor node is close in range. This would not only reduce excess power usage, but also reduce interference among nodes which leads to transmission failures. Another feature we plan to explore is a loop-detection algorithm to reduce the occurrence of packets being transmitted among a small subset of nodes that are disconnected from the rest of the network. Finally, we plan to investigate the inclusion of a MAC protocol with a more traditional handshaking scheme to replace the method employed by QELAR of simply overhearing transmissions and using this as an acknowledgment of transmission success. This could address issues such as false detections of packet failure that result in duplicate packet generation and therefore increased network utilization and energy usage.

\section{Acknowledgment}

This work is being funded under the Office of Naval Research (ONR) In-House Laboratory Research (ILIR) program at SSC Pacific.

\section{References}

[1] I. F. Akyildiz, D. Pompili, T. Melodia, State of the art in protocol research for underwater acoustic sensor networks, in: Proc. SIGMOBILE Mobile Computing Comm. Rev., volume 11, 2007, pp. 11-22. 
[2] R. Headrick, L. Freitag, Growth of underwater communication technology in the u.s. navy, in: IEEE Communications Magazine, 2009, pp. 80-82.

[3] J. Heidemann, M. Stojanovic, M. Zorzi, Underwater sensor networks: Applications, advances and challenges, in: Philos. Trans. R. Soc. A, volume 370, 2012, pp. 158-175.

[4] D. Pompili, I. F. Akyildiz, Overview of networking protocols for underwater wireless communication, IEEE. Commun. Mag., Feature Topic on Underwater Wireless Communications (2009).

[5] S. Climent, A. Sanchez, J. Capella, N. Meratnia, J. Serrano, Underwater acoustic wireless sensor networks: Advances and future trends in physical, mac and routing layers, Sensors 14 (2014) 795-833.

[6] P. Xie, J.-H. Cui, L. Lao, VBF: Vector-based forwarding protocol for underwater sensor networks, in: Proc. Networking, 2006, pp. 1216-1221.

[7] H. Yan, J. Shi, J.-H. Cui, DBR: Depth-based routing for underwater sensor networks, in: Proc. Networking, 2008, pp. $72-86$.

[8] Y. Chen, Y. Lin, Mobicast routing protocol for underwater sensor networks, in: Sensors, volume 13, 2013, pp. $737-749$.

[9] J. Jornet, M. Stojanovic, M. Zorzi, Focused beam routing protocol for underwater acoustic networks, in: Proc. ACM Int'l Workshop Underwater Networks, 2008, pp. 75-82.

[10] C.-H. Yang, K. Ssu, An energy-efficient routing protocol in underwater sensor netowrks, in: Proc. 3rd IEEE Int'l Conf. on Sensing Technology, Tainan, Taiwan, 2008, pp. 114-118.

[11] A. Wahid, L. Sungwon, K. Dongkyun, An energy-efficient routing protocol for uwsns using physical distance and residual energy, in: Proc. IEEE OCEANS 2011, Santander, Spain, 2011, pp. 1-6.

[12] W. Liang, H. Yu, L. Liu, B. Li, C. Che, Information-carrying based routing protocol for underwater acoustic sensor network, in: Proc. IEEE Int'l Conf. on Mechatronics and Automation, Harbin, China, 2007, pp. 729-734.

[13] L. Busoniu, R. Babuska, B. De Schutter, A compresive survey of multi-agent reinforcement learning, IEEE Trans. Syst., Man, Cybern. C: Appl. Rev. 38 (2008) 156-182.

[14] T. Hu, Y. Fei, QELAR: A machine-learning-based adaptive routing protocol for energy-efficient and lifetimeextended underwater sensor networks, IEEE Trans. Mobile Computing 9 (2010) 796-809.

[15] T. Hu, Y. Fei, Murao: A multi-level routing protocol for acoustic-optical hybrid underwater wireless sensor networks, in: 2012 9th Annual IEEE Communications Society Conference on Sensor, Mesh and Ad Hoc Communications and Networks, 2012, pp. 218-226.

[16] Ns-2, 2013. URL: http://nsnam.isi.edu/nsnam/index.php/.

[17] R. S. Sutton, A. Barto, Reinforcement Learning: An Introduction, The MIT Press, Cambridge, Massachusetts, 1998.

[18] M. Erol-Kantarci, H. Mouftah, S. Oktug, A survey of architectures and localization techniques for underwater acoustic sensor networks, IEEE Communications Surveys \& Tutorials 13 (2011) 487-502.

[19] M. Zorzi, P. Casari, N. Baldo, A. Harris III, Energy-efficient routing schemes for underwater acoustic networks, IEEE Journal on Selected Areas in Communications 26 (2008).

[20] W. Thorp, Analytic description of the lowfrequency attenuation coefficient, Journal of the Acoustical Society of America 42 (1967). 
[21] R. Coates, Underwater Acoustic Systems, John Wiley \& Sons, Inc., New York, 1989.

[22] R. E. Kalman, A new approach to linear filtering and prediction problems, in: Transactions of the ASME Journal of Basic Engineering, 1960, pp. 35-45.

[23] S. Kumar, R. Miikkulainen, Dual reinforcement q-routing: An on-line adaptive routing algorithm, in: Proc. Artificial Neural Networks in Eng. Conf., 1997, pp. 108-114.

[24] Desert, 2013. URL: http://nautilus.dei.unipd.it/desert-underwater.

[25] Woss, 2013. URL: http://telecom.dei.unipd.it/ns/woss/.

[26] Ns-miracle, 2013. URL: http://telecom.dei.unipd.it/pages/read/58/.

Randall Plate completed his Master of Science degree in Electrical Engineering at the University of Minnesota with an emphasis in digital signal processing in August of 2009. His research was focused on adaptive signal processing for the cancellation of feedback in digital hearing aids. He joined SSC-PAC in 2009 and began working in the areas of both acoustic and GPS navigation, including GPS-INS integration. His work in underwater acoustics includes active sonar processing, tracking algorithms, multi-modal communications, and acoustic data analysis.

Cherry Wakayama earned a Master of Science (2005) and a Doctor of Philosophy (2009) in Electrical Engineering from the University of Washington. She was awarded an Intel Foundation Ph.D. Fellowship for 2007-2009. She participated in several industry projects ranging from the modeling of three-dimensional via structures for high-speed data buses to the development of control algorithms for high-speed stock trading. Since joining SSCPAC in 2009, her technical focus has been on multistatic active sonar information fusion, sensor management, and field control algorithms. Her research interests include dynamical

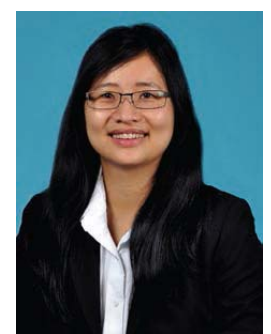
systems, optimal control theory and applications in operations research. 
Author Biographies (also present in manuscript ("AORUN-AdHocNetworksSpeciallssue.tex"))

Randall Plate completed his Master of Science degree in Electrical Engineering at the University of Minnesota with an emphasis in digital signal processing in August of 2009. His research was focused on adaptive signal processing for the cancellation of feedback in digital hearing aids. He joined SSC-PAC in 2009 and began working in the areas of both acoustic and GPS navigation, including GPS-INS integration. His work in underwater acoustics includes active sonar processing, tracking algorithms, multi-modal communications, and acoustic data analysis.

Cherry Wakayama earned a Master of Science (2005) and a Doctor of Philosophy (2009) in Electrical Engineering from the University of Washington. She was awarded an Intel Foundation Ph.D. Fellowship for 2007-2009. She participated in several industry projects ranging from the modeling of threedimensional via structures for high-speed data buses to the development of control algorithms for highspeed stock trading. Since joining SSC-PAC in 2009, her technical focus has been on multistatic active sonar information fusion, sensor management, and field control algorithms. Her research interests include dynamical systems, optimal control theory and applications in operations research. 



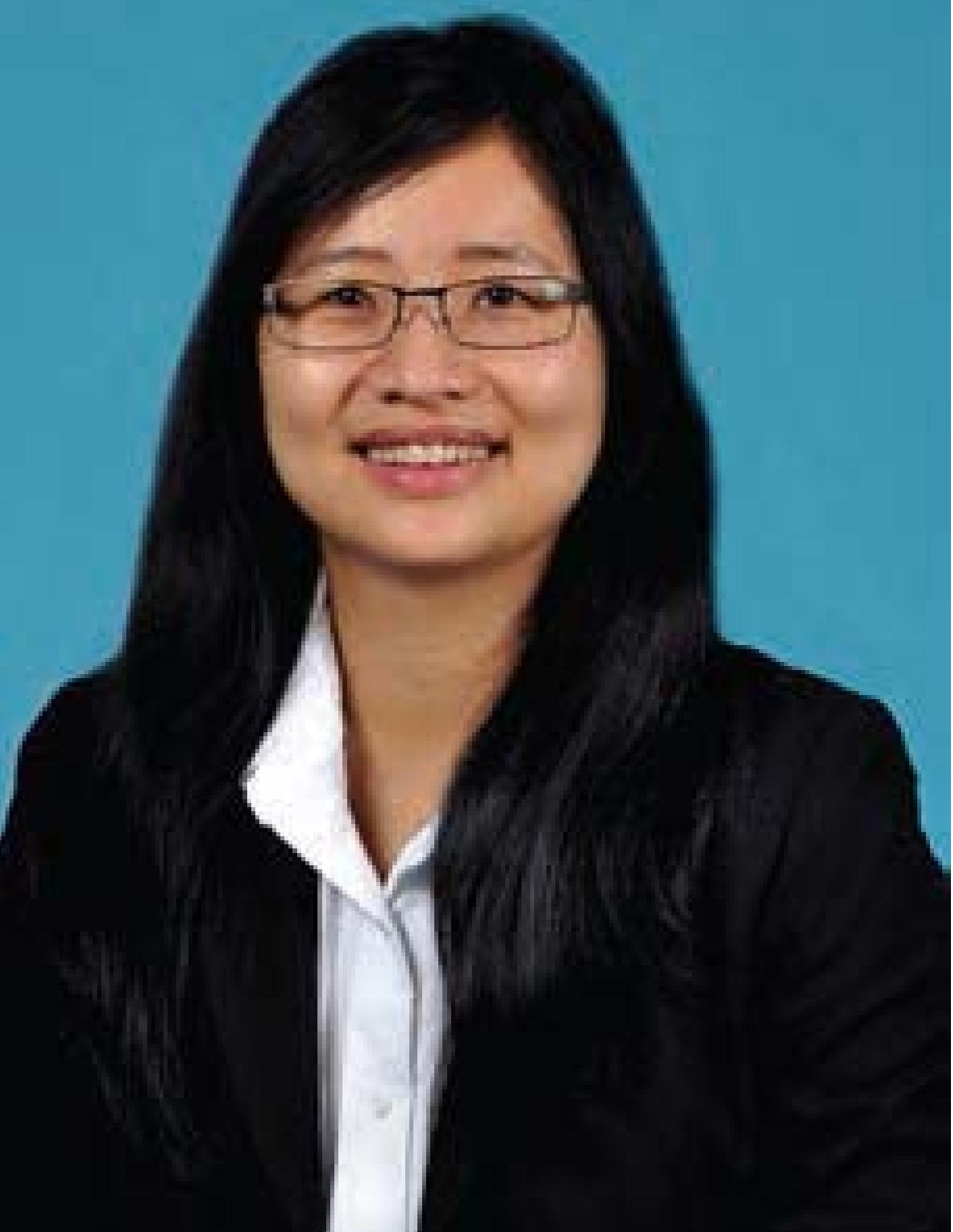

